\title{
Exploring Telomere Dynamics in Aging Male Rat Tissues: Can Tissue-Specific Differences Contribute to Age-Associated Pathologies?
}

\author{
Jane L. Tarry-Adkins ${ }^{\mathrm{a}, \mathrm{b}}$ Catherine E. Aiken ${ }^{\mathrm{a}} \mathrm{b} \quad$ Laura Dearden $^{\mathrm{a}}$ \\ Denise S. Fernandez-Twinn ${ }^{a}$ Susan Ozanne ${ }^{a}$ \\ aMetabolic Research Laboratories and MRC Metabolic Diseases Unit, Wellcome Trust-MRC Institute of \\ Metabolic Science, Addenbrooke's Hospital, University of Cambridge, Cambridge, UK; ${ }^{\text {b}}$ Department of \\ Obstetrics and Gynaecology, The Rosie Hospital and NIHR Cambridge Comprehensive Biomedical \\ Research Centre, University of Cambridge, Cambridge, UK
}

\section{Keywords}

Aging $\cdot$ Telomeres $\cdot$ Noncommunicable disease $\cdot$ Analysis

\begin{abstract}
Introduction: Due to increasing lifespan, global aging rates are rising rapidly and age-associated diseases are increasing. To ensure that health span is concomitant with life span, a greater understanding of cellular mechanisms of aging is important. Methods: Telomere length analysis from a wide range of tissues from weaning, young adult, and middleaged $(3,12$ and 52 week) male Wistar rats were conducted using Southern blotting. Telomere lengths were compared between tissues and ages using regression models based on the ratios of longest-to-shortest telomere fragments. $R \boldsymbol{e}$ sults: Robust linear age-dependent telomere attrition was observed in the liver; 3 versus 12 weeks, 3 versus 52 weeks $(p<0.01), 12$ versus 52 weeks $(p<0.05)$ and the heart; 3 versus 12 weeks $(p<0.05)$ and 3 versus 52 weeks $(p<0.001)$. More subtle shortening was observed in aorta and epididymal fat; 3 and 12 versus 52 weeks $(p<0.001)$ and in skeletal muscle; 3 versus 52 weeks ( $p<0.05), 12$ versus 52 weeks ( $p<$
\end{abstract}

0.01). Young thymus telomeres increased in length (3 vs. 12 weeks) and then shortened between 12 and 52 weeks ( $p<$ $0.001)$. We also reported disparity in telomere shortening within tissues: telomeres in aging brain cortex significantly shortened; 3 versus 52 weeks ( $p<0.05), 12$ versus 52 weeks $(p<0.01)$. This was not seen in the hypothalamic region. $\mathrm{A}$ robust stepwise shortening was observed in the renal cortex; 3 versus 12 weeks, 12 versus 52 weeks $(p<0.05)$, and 3 versus 52 weeks $(p<0.001)$, which was not as apparent in the renal medulla; 3 versus 12 weeks $(p<0.01)$ and 3 versus 52 weeks $(p<0.01)$. The vastus lateralis skeletal muscle demonstrated the shortest telomere length at weaning and underwent robust age-associated attrition; 3 versus 52 weeks ( $p<0.05), 12$ versus 52 weeks $(p<0.01)$. We demonstrated that specific tissues exhibit unique telomere attrition profiles which may partially explain why certain diseases are more prevalent in aged individuals. Discussion/Conclusion: We show wide variations between tissues in vulnerability to the aging process. In the future, this may help target potential interventions to improve health span.

(c) 2021 The Author(s) Published by S. Karger AG, Basel 


\section{Introduction}

The World Health Organization estimates that the number of people aged 65 years or older in the world will reach 1.5 billion in 2050, compared with 524 million in 2010 [1]. The aging population structure has contributed to get observed increases in age-associated diseases, including cardiovascular disease (CVD), type 2 diabetes (T2D), chronic obstructive pulmonary disease, cancers, dementia, renal dysfunction, nonalcoholic fatty liver disease, and obesity. These noncommunicable diseases (NCDs) are now responsible for $60 \%$ of all deaths world wide [2].

The increase in the aging population places an enormous strain on health care and social care systems throughout the world. Therefore, much research is focused on understanding the dynamics and underlying molecular mechanisms of the aging process. Age-associated disease prevalence is highly varied. For example, the so called "big four" NCDs (CVD, T2D, cancers, and chronic obstructive pulmonary disease) make up almost $30 \%$ of all NCDs, when expressed in the terms of disability-adjusted life year score (a measure of overall disease burden) [2]. A major caveat in dissecting out why these 4 NCDs are the most prevalent of all global NCDs, is in understanding how both modifiable environmental factors and inherent differences in the rate of cellular aging affects specific tissues. In order to address this question, studies of cellular aging mechanisms in a range of tissues are required.

Telomeres are guanine-rich repeat DNA sequences $\left(\mathrm{TTAGGG}^{n}\right)$ which are situated at the ends of chromosomes, and are essential to preserve genomic integrity and stability [3]. The extreme terminus of telomeres comprises a guanine-rich $3^{\prime}$ overhang that serves as a template for elongation, and forms a telomeric "T-loop" which acts to stabilze the telomere. Most healthy somatic cells do not express or have low levels of the telomere elongation enzyme, telomerase. Telomerase forms a protective "cap" around the telomere, thereby, preventing its shortening. It comprises 2 main components; Tert, a catalytic component with reverse transcriptase activity [4] and Terc, which serves as a template for telomeric DNA synthesis [5]. Without telomerase, the eukaryotic telomeres shorten with each cellular division, due to the $3^{\prime}$ end replication problem in which full extension of a linear chromosome cannot occur. When telomeres of somatic cells reach a critically short length, a conformational change that resembles a DNA damage occurs, and this triggers the cell senescence pathways. Senescent cells by nature are nonproliferating, and are known to contribute to aging-related diseases and mor- bidity [6]. Moreover, several studies have shown that telomere shortening is implicitly associated with cellular aging [7], disease pathogenesis [8, 9], and life span [10, 11]. Most importantly, it is evident that accumulation of critically short telomeres is essential in modulating the cell senescence and eventual disease pathogenesis [12]. Consequently, we developed a telomere length analysis methodology using a telomere restriction fragment (TRF) analysis in which the TRF smear was divided into 4 discreet regions based upon molecular weight. This included a very short size range (1.3-4.2 kb), intermediate short (4.2-8.6 kb), intermediate long (8.6-48.5 kb), and very long (48.5-145 $\mathrm{kb})$ telomere lengths [13].

Previously, we have demonstrated telomere shortening in several aging rat tissues (liver, lung, pancreas, and whole kidney), which was undetectable using the traditional mean whole telomere TRF method of telomere length analysis [13]. Whole brain, however, demonstrated no change in telomere length with age, using either of the methods [13]. In humans, it is apparent that an altered pattern of telomere length dynamics occurs along the life course. In the first years of life, a significant age-associated telomere shortening in leucocytes has been reported, albeit with a high variability reported between individuals [14]. Leukocyte telomere length, then, becomes relatively stable throughout later childhood, preadolescent, and adolescent years, and eventually, telomere shortening rate increases in very old age [15]. Moreover, telomere dynamics between the tissues may alter between tissues itself, which could potentially explain why different systems in the body are more vulnerable to age-associated changes than others.

This study, therefore, aimed to establish if telomere length in a wide range of rat tissues varied from an early timepoint (weaning) to 52 weeks of age. Second, we aimed to establish if different tissues exhibited altered rates of ageassociated telomere attrition. Within the experimental design, we performed both TRF length analysis methods, and further interrogated the size distribution analysis method to maximize its sensitivity. These data may identify tissues which are likely to be most vulnerable to external factors that influence the aging process, and therefore, this is why some NCDs are more prevalent than others.

\section{Materials and Methods}

Male and female Wistar rats (Rattus norvegicus) of breeding age (5-8 weeks of age) were purchased from Charles River, UK, which were allowed to acclimatise in specific pathogen-free (SPF) housing using individually ventilated cages with environmental enrichment, and then, mated to generate an in-house breeding
Tarry-Adkins/Aiken/Dearden/ Fernandez-Twinn/Ozanne 


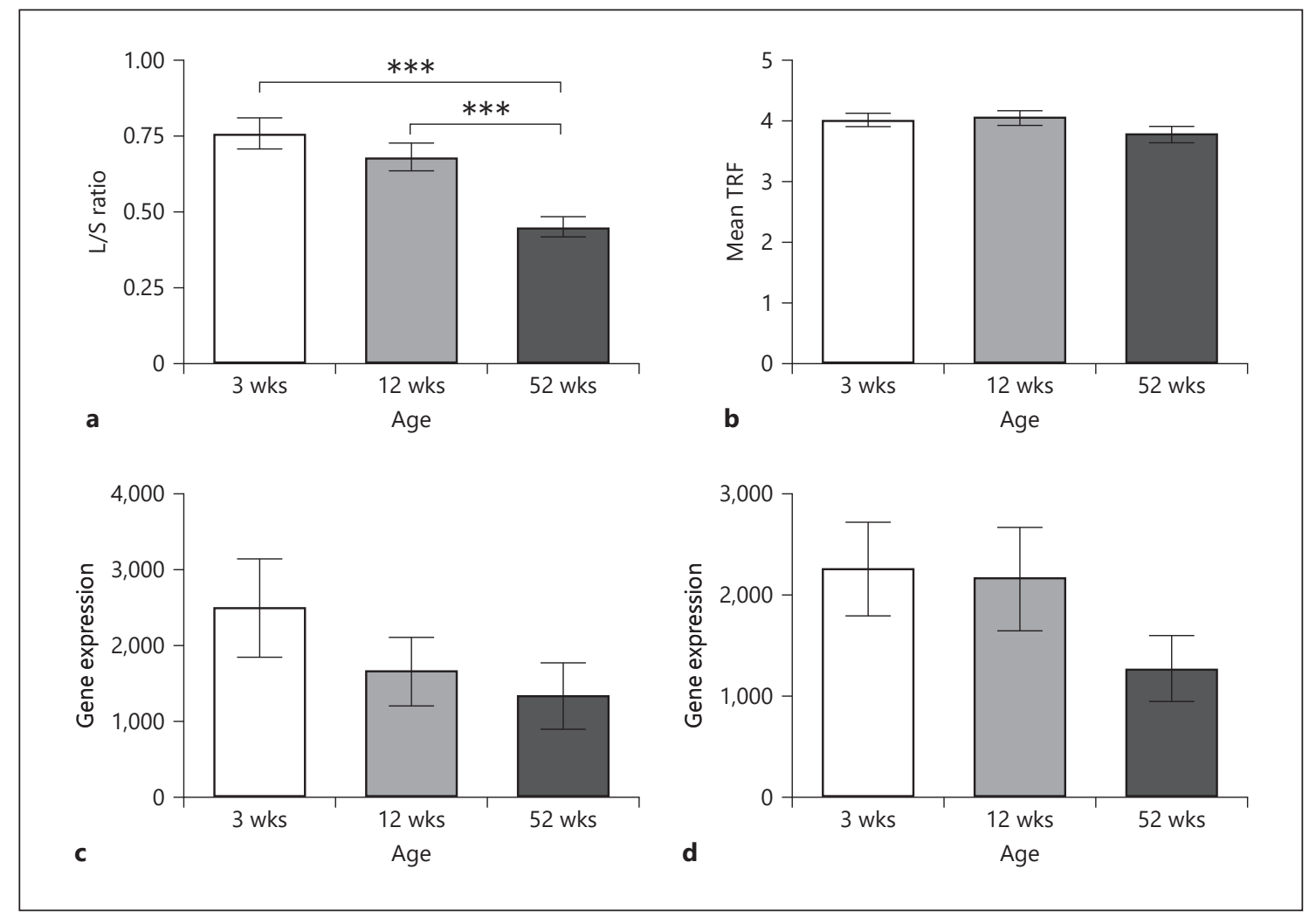

Fig. 1. The effect of aging upon overall telomere length measured by Southern blotting and telomerase expression by RT-PCR in rats at 3,12, and 52 weeks of age using discrete fragment TRF method (a) or Mean TRF method (b). Tert1 expression (c) and Terc expression (d). $N=8$ per group; \pm SEM. TRF, telomere restriction fragment.

Table 1. Primer table

\begin{tabular}{llll}
\hline Gene & Forward sequence & Reverse sequence & Product size, bp \\
\hline Tert1 & 5' CTGGCAGGTATACGGCTTTC 3' & 5' CTTTAAGAAGCGGCGCTCA 3' & 92 \\
Terc & 5' CCGCCGTGAAGAGCTAGT 3' & 5' TCAGGGACCAGTTCCGTTAC 3' & 97 \\
Ppia & 5' TGAGAACTTCATCCTGAAGCATACA 3' & 5' CATTTGTGTTTGGTCCAGCATT 3' & 89 \\
\hline
\end{tabular}

colony. Virgin female offspring weaned from the breeding colony were maintained at $22^{\circ} \mathrm{C}$, on a controlled 12:12-h light-dark cycle, and mated at approximately 6 weeks of age (around $240 \mathrm{~g}$ body weight). Pregnant rats were maintained at $22^{\circ} \mathrm{C}$, on a controlled 12:12-h light-dark cycle, in specific pathogen-free (SPF) housing using individually ventilated cages with environmental enrichment. Upon detection of vaginal plug, dams were maintained on a $20 \%$ protein control diet (consisting of $22 / 100 \mathrm{~g}$ casein protein [ $88 \mathrm{~g}$ protein/100 g], 5.05/100 g mineral and vitamin mixture, 0.22/100 g dI-methionine, 8/100 g maize starch, 5/100 g cellulose, $4.3 / 100$ g soyabean oil, and 55.15/100 g glucose) as previously described [16]. Access to the diet and water was provided ad libitum. The diet was purchased from Arie Blok (Woerden, The Netherlands). The day of birth was recorded as day 1 of postnatal life. Litters were standardized to 8 pups $48 \mathrm{~h}$ after birth, and 4 males in each litter were used in this study. Animals were weaned at 21 days of age onto a standardized pellet diet (RM3) (consisting of $15.43 \%$ fiber, $1.43 \%$ pectin, $9.2 \%$ hemicellulose, $3.93 \%$ cellulose, $1.5 \%$ lignin, $33.92 \%$ starch, $5.75 \%$ sugar, $20.77 \%$ assorted amino acids, $4.54 \%$ assorted fatty acids, and $4.65 \%$ macro minerals (Special Diet Services, Witham, Essex, UK). One male in each litter was culled at 3 weeks of age; 1 at 12 weeks of age, and a third male was culled at 52 weeks of age. All animals were killed by $\mathrm{CO}_{2}$ asphyxiation at approximately 10:00 a.m. after an overnight fast. At postmortem, the heart, aorta, liver, kidney cortex, kidney medulla, vastus lateralis (VL) skeletal muscle, liver, epididymal fat, thymus, spleen, and brain were removed, weighed, and snap-frozen in liquid nitrogen and then stored at $-80^{\circ} \mathrm{C}$ until analysis. The brain was dissected into cortex and hypothalamic regions after freezing. In all cases, $n$ refers to the number of litters used with the mother as the statistical unit and with 1 animal used from each litter at each time point. 
Telomere Length Measurement

High molecular weight genomic DNA was extracted using a Phenol/Chloroform DNA methodology. DNA quantity and purity were determined using a NanoDrop spectrophotometer (Nanodrop Technologies, Wilmington, DE, USA) [17]. DNA integrity was confirmed by the agarose gel electrophoresis. DNA $(1.2 \mu \mathrm{g})$ was digested by Hinfl and Rsal restriction enzymes at $37^{\circ} \mathrm{C}$ for $2 \mathrm{~h}$, separated by the pulsed field gel electrophoresis, and transferred to nylon membranes by Southern blotting $[17,18]$. Standard undigested and digested genomic DNA from a 3-week animal was also included on each gel to verify digestion efficiency as described previously [17]. Telomere length was measured using Telo TAGGG telomere length assays (Roche Diagnostics, Welwyn Garden City, Herts, UK). Telomere signals were analyzed using Alpha Ease Software (Alpha Innotech, Exeter, Devon, UK). Telomere length was measured using the mean TRF methodology, in which the whole telomere smear was quantified. This methodology is detailed in the manufacturers' instructions (Roche Diagnostics, Welwyn Garden City, Herts, UK). This was compared with our method of "discrete fragment TRF" telomere length analysis, in which the percentage intensity (\% telomere length) of the telomeric signal was determined according to 4 molecular size regions, defined by molecular weight markers $[17,18]$.

\section{Telomere Length Analysis}

Telomere length analysis was performed based on the telomere restriction fragments (TRF). We performed a head to head analysis of methods based on the mean TRF, in which the whole telomere smear was quantified, and analysis in which the TRF smear was divided into 4 discreet regions. The 4 regions were: very short (1.3-4.2 kb), intermediate short $(4.2-8.6 \mathrm{~kb})$, intermediate long (8.6-48.5 kb), and very long (48.5-145 kb) and were defined by molecular weight markers $[13,17,18]$.

In the initial modeling phase, we compared 4 different ways of expressing telomere length. These were (i) the mean TRF method, (ii) the weighted mean percentage telomere fragment length in each sample (i.e., the index of all molecular weight "bins" together), (iii) the percentage of telomeres falling within each fragment length bin separately, and (iv) the ratio of the percentage of very long/very short telomeres (\% L/S). We used linear regression models including variables for age and tissue type to compare the model fit for each method. The optimal model was selected using the Akaike Information Criterion and lowest residual values. Using the ratio of long-to-short telomeres (\% L/S) gave the best fit to the overall data, particularly when compared with the mean TRF method that is more often used [19]. The \% L/S method could detect an overall telomere shortening of combined tissues (shown in Fig. 1a), which was not possible using the mean TRF method (shown in Fig. 1b).

Gene Expression of Components Modulating Telomere Length RNA was extracted using an RNeasy Plus Mini Kit (Qiagen, Hilden, Germany) following the manufacturers' instructions. A DNase digestion step was performed in order to ensure no gDNA contamination. RNA $(1 \mu \mathrm{g})$ was used to synthesize cDNA using oligo-dT primers and M-MLV reverse transcriptase (Promega, Southampton, Hants, UK). Gene expression was determined using custom-designed primers (Sigma, Poole, Dorset, UK) and SYBR Green reagents (Applied Biosystems, Warrington, Cheshire, UK). Primer sequences are presented in Table 1. Quantification of gene expression was performed using a Step 1 Plus RT-PCR machine
Table 2. Morphometric analysis of aging male rats

\begin{tabular}{lccc}
\hline Tissue & 3 weeks & 12 weeks & \multicolumn{1}{l}{52 weeks } \\
\hline Body weight, g & $49 \pm 2^{* * *}$ & $475 \pm 15^{\$ \$}$ & $956 \pm 41^{\&}$ \\
Aorta, mg & $11 \pm 1^{* * *}$ & $63 \pm 11^{\$ \$}$ & $85 \pm 7^{\& \& \&}$ \\
Heart, mg & $37 \pm 2^{* * *}$ & $1,436 \pm 85^{\$ \$}$ & $2,055 \pm 164^{\& \& \&}$ \\
Kidney (L), mg & $30 \pm 1^{* * *}$ & $1,800 \pm 63^{\$ \$}$ & $2,533 \pm 163^{\& \& \&}$ \\
Liver, mg & $178 \pm 7^{* * *}$ & $17,345 \pm 758^{\$ \$}$ & $28,608 \pm 2,041^{\& \& \&}$ \\
Thymus, mg & $12 \pm 1^{* * *}$ & $753 \pm 55^{\$ \$}$ & $1,197 \pm 96^{\& \& \&}$ \\
Spleen, mg & $26 \pm 4^{* * *}$ & $1,129.0 \pm 67^{\$ \$ \$}$ & $1,735 \pm 116^{\& \& \&}$ \\
Brain, mg & $144 \pm 2^{* * *}$ & $2,050 \pm 34^{\$ \$}$ & $2,181 \pm 38^{\& \&}$ \\
VL, mg & $45 \pm 11^{* * *}$ & $4,115 \pm 145^{\$ \$}$ & $6,691 \pm 373^{\& \& \&}$ \\
Epi fat, mg & - & $8,396 \pm 895$ & $23,458 \pm 2,206^{\& \& \&}$ \\
\hline
\end{tabular}

VL, vastus lateralis. ${ }^{* * *} p<0.001,3$ versus 12 weeks. ${ }^{\$ \$} p<$ $0.001,12$ versus 52 weeks. ${ }^{\&} p<0.05,{ }^{\& \&} p<0.01,{ }^{\& \& \&} p<0.001$, 3 versus 52 weeks.

(Applied Biosystems, Warrington, Cheshire, UK). Equal efficiency of the reverse transcription of RNA from all groups was confirmed through quantification of expression of the housekeeping gene Ppia. Expression of Ppia did not differ with respect to age for any tissues studied (online suppl. Table 1; for all online suppl. material, see www.karger.com/doi/10.1159/000511608).

\section{Statistical Analysis}

Statistical analysis for telomere length and gene expression was conducted using a one-way ANOVA with age as the independent variable and Duncan's post hoc testing where appropriate, using Statistica (Statsoft, Palo Alto, CA, USA). Logistic regression and correction for multiple hypothesis testing was performed using the $R$ version 3.6.2 (R Foundation for Statistical Computing, Vienna, Austria). Wherever $p$ values are reported, an alpha level $<0.05$ was considered statistically significant. Data are represented as mean \pm SEM.

\section{Results}

\section{Morphometric Analysis of Aging Male Rat Tissues}

As expected, the body weights and all tissue weights increased significantly with age (Table 2 ).

\section{Overall Effect of Aging upon Telomere Length in Male Rat Tissues}

Telomere length decreased $(p<0.001)$ with age when assessed using the ratio of long-to-short telomeres (shown in Fig. 1a), but this was not apparent using the mean TRF method (shown in Fig. 1b).

\section{Overall Effect of Aging upon Telomerase Expression}

Although overall Tert 1 and Terc expression numerically decreased with age, this was statistically non-significant $($ Tert $1 ; p=0.24)(\operatorname{Ter} c ; p=0.22)$ (shown in Fig. $1 c, d)$. 


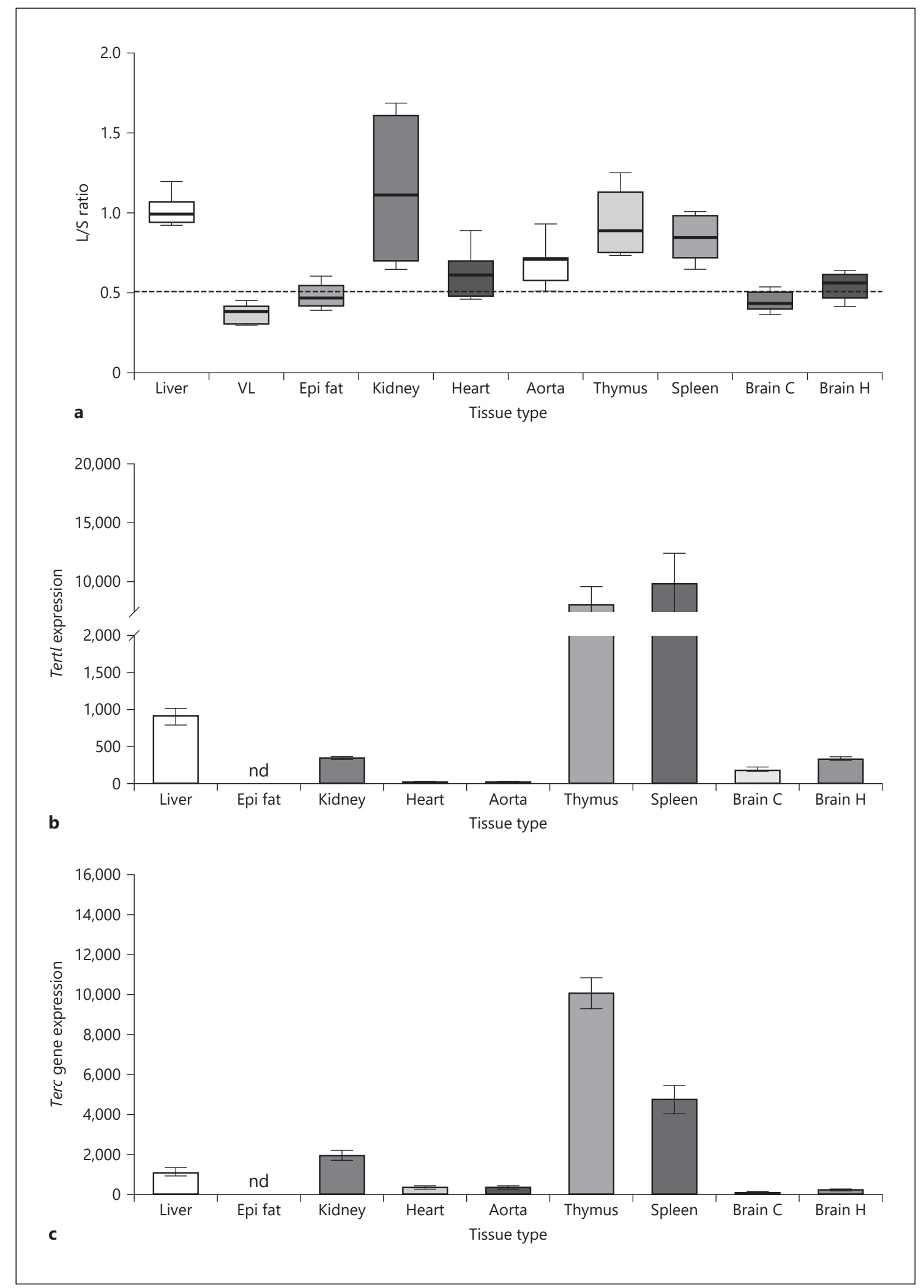

Fig. 2. Telomere length measured by Southern blotting (a), Tert1 expression (b) and Terc expression (c) measured by RT-PCR in weanling male rats. $N=8$ per group; \pm SEM. VL, vastus lateralis. 
The Effect of Tissue Type upon Telomere Length in Male Rats at Weaning

At weaning, the telomere length varied by tissue type: The ratio of long-to-short telomeres was highest (approximately $1.0 \% \mathrm{~L} / \mathrm{S})$ in the kidney $(1.14 \pm 0.2 \% \mathrm{~L} / \mathrm{S})$, the liver $(1.01 \pm 0.04 \% \mathrm{~L} / \mathrm{S})$, and thymus $(0.93 \pm 0.08 \% \mathrm{~L} / \mathrm{S})$ (shown in Fig. 2a). This was followed by telomere lengths in the spleen $(0.73 \pm 0.13 \% \mathrm{~L} / \mathrm{S})$, heart $(0.61 \pm 0.07 \% \mathrm{~L} / \mathrm{S})$ and aorta $(0.68 \pm 0.05 \% \mathrm{~L} / \mathrm{S})$, the epididymal fat pad $(0.48$ $\pm 0.03 \% \mathrm{~L} / \mathrm{S})$, brain cortex $(0.44 \pm 0.02 \% \mathrm{~L} / \mathrm{S})$, and brain hypothalamus $(0.54 \pm 0.03 \% \mathrm{~L} / \mathrm{S})$ (shown in Fig. 2a). Telomeres from the VL skeletal muscle were shortest $(0.36 \pm 0.02 \% \mathrm{~L} / \mathrm{S})$ (shown in Fig. 2a).

\section{Telomerase Components at Weaning}

Different tissues had a range of telomerase expression levels at weaning. Detectable levels of Tert1 and Terc was observed in the liver of weanling rats, with lower but still detectable expression of telomerase observed in the kidney, which was also the case for Tert 1 expression in brain cortex and hypothalamus (shown in Fig. 2b, c). The heart and aorta were on the margins of detection for telomerase expression. Tert 1 and Terc were not detectable in epididymal fat (shown in Fig. 2b, c). The immunological tissues (thymus and spleen) had an extremely high expression of both Tert 1 and Terc (shown in Fig. 2b, c) at weaning.

\section{Comparison of Age-Associated Telomere Length Dynamics}

A significant age-associated telomere attrition was observed in the classical insulin sensitive tissues (except brain and kidney): liver ( 3 vs. 12 weeks; $p<0.01,12$ vs. 52 weeks; $p<0.05$, 3 vs. 52 weeks; $p<0.01$ ), VL ( 3 vs. 52 weeks; $p<0.05,12$ vs. 52 weeks; $p<0.01$ ), and the epididymal fat pad ( 3 and 12 vs. 52 weeks; $p<0.001$ ) (shown in Fig. 3a).

Telomeres from both regions of the kidney shortened significantly with age: kidney cortex ( 3 vs. 12 weeks; $p<$ $0.05,12$ vs. 52 weeks; $p<0.05$ and 3 vs. 52 weeks; $p<$ 0.001 ) (shown in Fig. 3b) and kidney medulla (3 vs. 12 weeks and 3 vs. 52 weeks, $p<0.01$ ) (shown in Fig. 3b). In the cardiovascular system, significant age-associated telomere shortening was observed in both cardiac ( 3 vs. 12 weeks; $p<0.05$ and vs. 52 weeks, $p<0.001$ ) (shown in Fig. 3c) and aortic tissues ( 3 and 12 vs. 52 weeks; $p<$ 0.001) (shown in Fig. 3c).

Immunological tissues demonstrated a unique pattern of telomere length dynamics. In the thymus, telomere length elongated significantly in the young tissue then between 12 and 52 weeks decreased significantly back to levels observed at 3 weeks ( 3 vs. 12 weeks and vs. 52 weeks; $p<0.001$ ) (shown in Fig. 3d). In the spleen, no significant age-associated telomere shortening was observed (shown in Fig. 3d). The brain cortex demonstrated shorter telomeres at 52 weeks compared to 3 weeks $(p<0.05)$ and compared to 12 weeks $(p<0.01)$ (shown in Fig. 3e); however, no change in telomere length was observed in the hypothalamic region of the brain (shown in Fig. 3e).

\section{Discussion}

Improved understanding of mechanisms that modulate aging may ultimately lead to the extension of health span. Telomere length is a robust marker of cellular aging and disease pathogenesis $[7,11]$ and is often considered a proxy for biological aging.

We demonstrate the significant shortening of telomeres in aging tissues which were not detected using the mean TRF method. Our mathematical modeling suggests that measurement of discrete areas of the telomere is the optimum method of telomere length measurement. These findings are particularly important, given that the loss of a few hundred base pairs from short telomeres is pivotal to cellular aging $[11,12]$. Such losses may go undetected by a traditional mean TRF analysis as the TRFs with few telomeric repeats seem to be obscured by the strong signal from other TRFs with long telomeres.

Weaning Telomere Length and Telomerase Expression

Telomeres at weaning were longest in the thymus, liver, and kidney. In the thymus, preservation of telomere length is essential to preserve immunological function and T-cell lineage [20] and may be related to the high expression of telomerase in young thymic tissue, as evidenced in this study and in previous work [20,21]. In rats, longer hepatic telomeres at weaning may also relate in part to the marginally telomerase-positive nature of this tissue [22]. This is recapitulated by the observed low, but detectable expression of Tert 1 and Terc mRNA. The liver is a regenerative organ and telomerase expression has been linked with the tissue's regenerative properties [23, 24]. Therefore, rat hepatic telomeres may start longer in early postnatal life to prepare for the potential increased cell division later in life when the liver may need to regenerate. Male rat's kidneys are known to deteriorate relatively quickly with age and have increased susceptibility to age-associated end-stage renal damage, with renal disease the most common cause of mortality in aging male rats [25]. Therefore, long renal telomeres at weaning may 

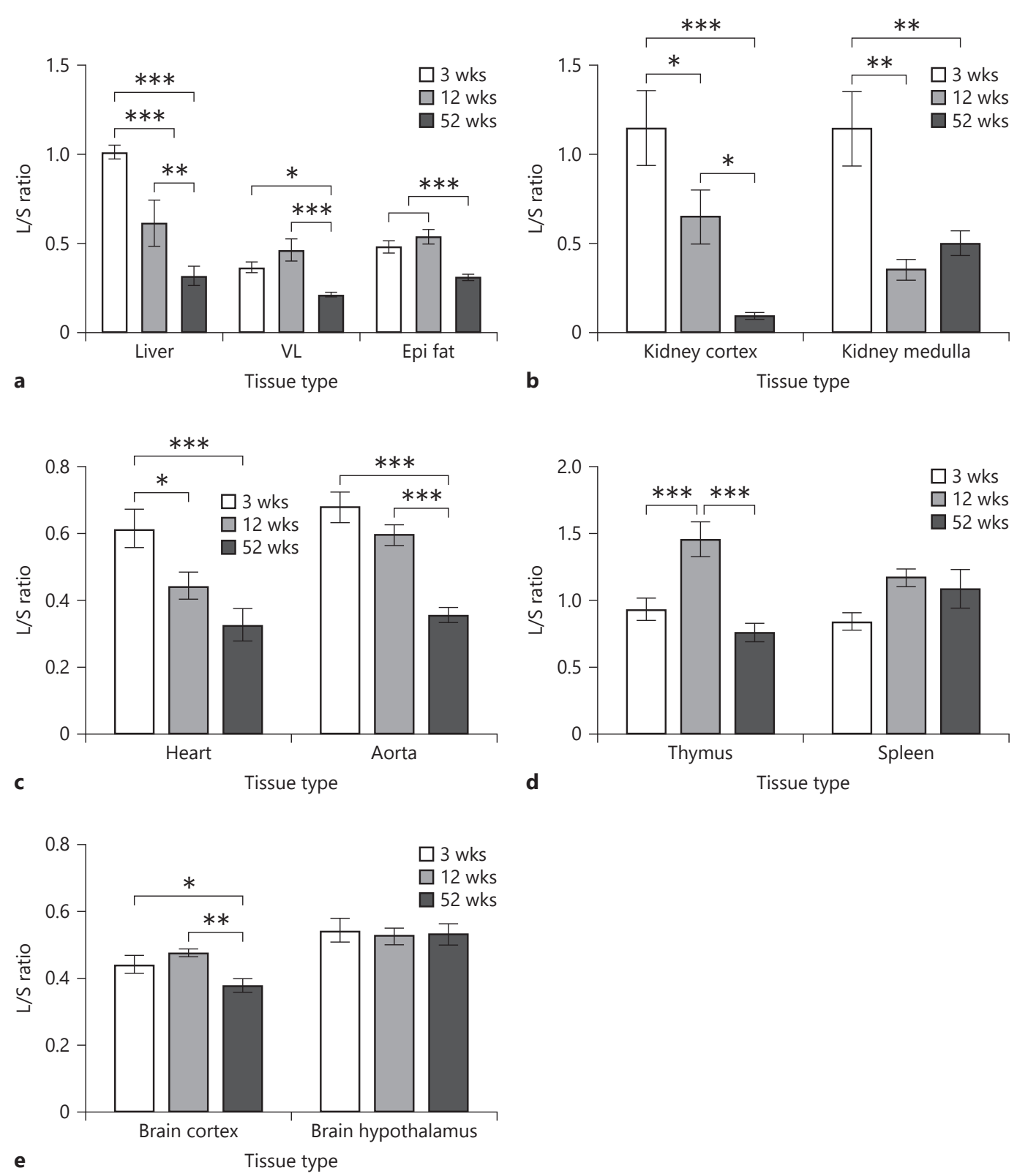

Fig. 3. The effect of individual tissue types upon telomere length measured by Southern blotting in: insulin sensitive (a), urinary system (b), cardiovascular system $(\mathbf{c})$, immunological system (d), brain cortex and hypothalamus (e). $N=8$ per group; \pm SEM. VL, vastus lateralis.

be a compensatory attempt to preserve telomere length and cellular integrity as long as possible. Preservation of renal telomere length at weaning may be related to the detectable levels of Tert 1 and Terc expression. In contrast, VL telomeres at weaning demonstrated the smallest proportion of long telomeres suggesting that telomeres in this type of skeletal muscle are already much shorter than in other tissues in early life.

\section{Tissue-Specific Telomere Length}

The rates and patterns of age-associated changes in telomere length differed between tissues. All classical in- 
sulin sensitive tissues with the exception of the brain underwent significant telomere shortening with age: The liver underwent a significant step wise shortening (behaving like a classical somatic cell population). Accelerated hepatic telomere shortening is associated with the progression of liver disease including nonalcoholic fatty liver disease, fibrosis [26], cirrhosis, and hepatocellular carcinoma [27]. The epididymal fat maintained longer telomeres at 3 and 12 weeks of age, which then drops off significantly at 52 weeks of age. In VL, the robust age-associated telomere shortening combined with the fact that this tissue started with the shortest telomeres at weaning may have particular detrimental implications for the etiology of glucose intolerance, insulin sensitivity, and T2D [28-30].

Subtle differences in the dynamics of age-associated telomere shortening were apparent between regions of the kidney. The renal cortex underwent a robust stepwise shortening with significant telomere attrition observed throughout the aging process. However, the renal medulla underwent a more graded telomere attrition which occurred only between young (3-12 weeks of age) tissues. Previously, we demonstrated that the cortex region of rat kidney shortens faster than the medulla [31], which is also reflective of that observed in the human [32]. This may potentially be due to the differences in mitochondrial density. Mammalian proximal renal tubules contain an abundance of large mitochondria dependent on oxidative rather than anaerobic metabolism [33]. In contrast, the cells within the inner medulla are known to have a far lower mitochondrial content than the cortical cells. Mitochondria are known contributors to reactive oxygen species (ROS) generation [34], which preferentially damages telomeric DNA sequences, due to guanine-rich residues within the telomere [35]. The renal cortex may be more liable to generate ROS via oxidative metabolism than the more anaerobic medullary cells and therefore will have telomeres which shorten faster than those found in the medulla.

We also observed an age-associated shortening of cardiac telomeres, suggesting that this tissue is behaving like a classic somatic cell type. Aortic telomeres also shorten with age; however, this began after 12 weeks, with no significant shortening observed in young tissue. Cardiac tissue is extremely metabolically active, and cardiomyocytes have a high mitochondrial mass by comparison to other less metabolically active tissues [36]. Conversely, myocytes from smooth muscle (aorta) have relatively few mitochondria $(22 \%$ of the estimated number in cardiac muscle) [37]. Therefore, it is plausible that the increased mitochondrial mass in cardiac muscle is a key modulator of the accelerated telomere shortening observed in the heart.

Between 3 and 12 weeks of age, the ratio of long-toshort telomeres in the thymus increased. This pattern was unique to this tissue, and may be necessary for the development of immunological function [20]. We previously reported that telomere length in the brain did not change in the aging male rat [13], which has also been reported in human brain tissue [38]. We also observed that age-associated telomere length attrition was much less pronounced in the brain tissue overall compared with other tissues. Interestingly, when the cortex and hypothalamic regions were analyzed separately, the telomere shortening was detected in the cortex which was not observed in the hypothalamus. It has been demonstrated that the cortical brain regions are more sensitive to development of Alzheimer's disease [39]. Therefore, the observed accelerated telomere shortening in the brain cortex may be a driving factor in its susceptibility to age-associated neurodegeneration which specifically affect cortical regions of the brain.

In conclusion, our findings are consistent with factors relating with telomere length dynamics being pivotal in cell senescence, and that the observed patterns vary considerably by tissue type, [40]. Telomere length in early postnatal life (weaning) could be regarded as an important setpoint in determining the fate of the tissue in later life, particularly if this is combined with a high rate of telomere length attrition as demonstrated in skeletal muscle. This is particularly important given recent evidence suggesting that the rate of telomere attrition is a powerful predictor of life span [11]. However, rapid telomere attrition may occur irrespective of the initial "setpoint" of telomere length as demonstrated in cardiac tissue, and in the kidney in which telomeres were not short at weaning; however, they underwent robust rates of age-associated telomere attrition. High mitochondrial density and oxidative stress are, perhaps, pivotal mechanisms for these findings. Our findings may partially explain why CVD and T2D are common NCDs; however, we acknowledge that we cannot claim causality based upon these findings alone. We also demonstrated that the function of the tissue may also be a factor in determining telomere dynamics: The urinary tract, CNS, and immunological tissues demonstrate markedly different age-associated telomere dynamics, potentially driven by the differences in cell proliferation and/or different mitochondrial densities, yet, this association needs to be fully elucidated. A greater understanding of telomere dynamics could potentially help determine which tissues are more vulnerable to the aging process and
Tarry-Adkins/Aiken/Dearden/ Fernandez-Twinn/Ozanne 
help to target any potential interventions. We also demonstrated that our discrete TRF telomere fragment analysis was the more sensitive and reliable approach for analyzing telomere length in a wide range of aging rat tissues compared with the mean TRF method in a comprehensive range of rat tissues.

\section{Statement of Ethics}

All protocols were approved by the Animal Welfare and Ethical Review Board (AWERB) of the University of Cambridge and carried out in accordance with the UK Animals (Scientific Procedures) Act 1986 and followed the ARRIVE guidelines.

\section{Conflict of Interest Statement}

The authors have no conflicts of interest to declare.

\section{Funding Sources}

J.L.A., S.E.O., and D.S.F.T. are funded by the British Heart Foundation (RG/17/12/33167). J.L.A., S.E.O. (MC_UU_00014/4), and D.S.F.T. (MC_UU_12012/4) are also funded by the Medical Research Council. C.E.A. is supported by an Action Medical Research grant (GN2788). L.D. is a Sir Henry Wellcome Post-Doctoral Fellow $(106026 / \mathrm{Z} / 14 / \mathrm{Z})$.

\section{Author Contributions}

J.L.A.: conceptualization, data curation, formal analysis, investigation, methodology, and writing (original draft \& review and editing). C.E.A.: investigation, methodology, validation, visualization, and writing (original draft \& review and editing). L.D.: resources and writing (original draft \& review and editing). D.S.F.T.: resources and writing (original draft \& review and editing). S.E.O.: conceptualization, funding acquisition, project administration, supervision, and writing (original draft \& review and editing).

\section{References}

1 Tarry-Adkins JL, Ozanne SE. Nutrition in early life and age-associated diseases. Aging Res Rev. 2017 Oct;39:96-105.

2 Lopez AD, Williams TN, Levin A, Tonelli M, Singh JA, Burney PG, et al. Remembering the forgotten non-communicable diseases. BMC Med. 2014 Oct;12(200):200-19.

3 Blackburn EH, Szostak JW. The molecular structure of centromeres and telomeres. Annu Rev Biochem. 1984 Jul;53:163-94.

4 Lingner J, Hughes TR, Shevchenko A, Mann M, Lundblad V, Cech TR. Reverse transcriptase motifs in the catalytic subunit of telomerase. Science. 1997 Apr;276(5312):561-7.

5 Feng J, Funk WD, Wang SS, Weinrich SL, Avilion AA, Chiu CP, et al. The RNA component of human telomerase. Science. 1995 Sep; 269(5228):1236-41.

$6 \mathrm{He} \mathrm{S}$, Sharpless NE. Senescence in health and disease. Cell. 2017 Jun;169(6):1000-11.

7 Blasco MA. Telomeres and human disease: aging, cancer and beyond. Nat Rev Genet. 2005 Aug;6(8):611-22.

8 Haycock PC, Heydon EE, Kaptoge S, Butterworth AS, Thompson A, Willeit P. Leucocyte telomere length and risk of cardiovascular disease: systemic review and meta-analysis. BMJ. 2014 Jul;8(349):g4227.

9 Zhao J, Miao K, Wang H, Ding H, Wang DW. Association between telomere length and type 2 diabetes mellitus: a meta-analysis. PLoS One. 2013 Nov;8(11):e79993.

10 Cawthorn RM, Smith KR, O'Brien E, Sivatchenko A, Kerber RA. Association between telomere length in blood and mortality in people aged 60 years or older. Lancet. 2003 Feb; 361(9355):393-5.
11 Whittemore K, Vera E, Martínez-Nevado E, Sanpera C, Blasco MA. Telomere shortening rate predicts species life span. Proc Natl Acad Sci U S A. 2019 Jul;116(30):15122-7.

12 Hemann MT, Strong MA, Hao LY, Greider $\mathrm{CW}$. The shortest telomere, not average telomere length, is critical for cell viability and chromosome stability. Cell. 2001 Oct;107(1): 67-77.

13 Cherif H, Tarry JL, Ozanne SE, Hales CN. Aging and telomeres: a study into organ- and gender-specific telomere shortening. Nucleic Acids Res. 2003 Mar;31(5):1576-83.

14 Rufer N, Brümmendorf $\mathrm{TH}$, Kolvraa $\mathrm{S}$, Bischoff C, Christensen K, Wadsworth L, et al. Telomere fluorescence measurements in granulocytes and $\mathrm{T}$ lymphocyte subsets point to a high turnover of hematopoietic stem cells and memory T cells in early childhood. J Exp Med. 1999 Jul;190(2):157-67.

15 Oeseburg H, de Boer RA, van Gilst WH, van der Harst P. Telomere biology in healthy aging and disease. Pflugers Arch. 2010 Jan; 459(2):259-68

16 Snoeck A, Remacle C, Reusens B, Hoet JJ. Effect of a low protein diet during pregnancy on the fetal rat endocrine pancreas. Biol Neonate. 1990;57(2):107-18.

17 Tarry-Adkins JL, Martin-Gronert MS, Chen JH, Cripps RL, Ozanne SE. Maternal diet influences DNA damage, aortic telomere length, oxidative stress, and antioxidant defense capacity in rats. FASEB J. 2008 Jun; 22(6):2037-44
18 Tarry-Adkins JL, Ozanne SE. Telomere length analysis: a tool for dissecting aging mechanisms in developmental programming. In. Investigations of early nutrition effects on long-term health. Methods in molecular biology. New York, NY: Humana Press; 2018. Vol. 1735.

19 Kimura M, Stone RC, Hunt SC, Skurnick J, Lu $\mathrm{X}$, Cao X, et al. Measurement of telomere length by the southern blot analysis of terminal restriction fragment lengths. Nat Protoc. 2010 Sep;5(9):1596-607.

20 Weng NP, Hathcock KS, Hodes RJ. Regulation of telomere length and telomerase in $\mathrm{T}$ and $B$ cells: a mechanism for maintaining replicative potential. Immunity. 1998 Aug;9(2): 151-7.

21 Tarry-Adkins JL, Aiken CE, Ashmore TJ, Fernandez-Twinn DS, Chen JH, Ozanne SE. A suboptimal maternal diet combined with accelerated postnatal growth results in an altered aging profile in the thymus of male rats. FASEB J. 2019 Jan;33(1):239-53.

22 Yamaguchi Y, Nozawa K, Savoysky E, Hayakawa N, Nimura Y, Yoshida S. Change in telomerase activity of rat organs during growth and aging. Exp Cell Res. 1998 Jul; 242(1):120-7.

23 Golubovskaya VM, Presnell SC, Hooth MJ, Smith GJ, Kaufmann WK. Expression of telomerase in normal and malignant rat hepatic epithelia. Oncogene. 1997 Sep;15(10): 1233-40.

24 Wege H, Brummeldorf TH. Telomerase activation in liver regeneration and hepatocarcinogenisis: Dr. Jekyl or Mr. Hyde? Curr Stem Cell Res Ther. 2007 Jan;2(1):31-8. 
25 Iwasaki K, Gleiser CA, Masoro EJ, McMahan CA, Seo EJ, Yu BP. The influence of dietary protein source on longevity and age-related disease processes of Fischer rats. J Gerontol. 1988 Jan;43(1):B5-12.

26 Donati B, Valenti L. Telomeres, NAFLD and chronic liver disease. Int J Mol Sci. 2016 Mar; 17(3):383.

27 Kitada T, Seki S, Kawakita N, Kuroki T, Monna $\mathrm{T}$. Telomere shortening in chronic liver diseases. Biochem Biophys Res Commun. 1995 Jun;211(1):33-9.

28 Ahmed S, Heraclides A, Sun Q, Elgzyri T, Ronn $\mathrm{T}$, Ling $\mathrm{C}$, et al. Telomere length in blood and skeletal muscle in relation to measures of glycaemia and insulinaemia. Diabet Med. 2012 Oct;29(10):377-81.

29 Gardner JP, Li S, Srinivasan SR, Chen W, Kimura M, Lu X, et al. Rise in insulin resistance is associated with escalated telomere attrition. Circulation. 2005 May;111(17):2171-7.

30 Adaikalakoteswari A, Balasubramanyam M, Mohan V. Telomere shortening occurs in Asian Indian type 2 diabetic patients. Diabet Med. 2005 Sep;22(9):1151-6.
31 Tarry-Adkins JL, Ozanne SE, Norden A, Cherif $\mathrm{H}$, Hales $\mathrm{CN}$. Lower antioxidant capacity and elevated p53 and p 21 may be a link between gender disparity in renal telomere shortening, albuminuria, and longevity. Am J Physiol Renal Physiol. 2006 Feb;290(2):F50916.

32 Melk A, Ramassar V, Helms LM, Moore R, Rayner D, Solez K, et al. Telomere shortening in kidneys with age. J Am Soc Nephrol. 2000 Mar;11(3):444-53.

33 Maunsbach AB, Christensen EI. Functional ultrastructure of the proximal tubule. In Handbook of physiology. Renal physiology. Bethesda, MD: American Journal of Physiology Society; 1992. Sect. 8, Vol. I, chapt. 2. p. 41-108.

34 Murphy MP. How mitochondria produce reactive oxygen species. Biochem J. 2009 Jan; 417(1):1-13.
35 Von Zglinicki T. Oxidative stress shortens telomeres. Trends Biochem Sci. 2002 Jul; 27(7):339-44.

36 Robin ED, Wong R. Mitochondrial DNA molecules and virtual number of mitochondria per cell in mammalian cells. J Cell Physiol. 1988 Sep;136(3):507-13.

37 Park S-Y, Gifford JR, Andtbacka RHI, Trinity JT, Hyngstrom JR, Garten RS, et al. Cardiac, skeletal and smooth muscle mitochondrial respiration: are all mitochondria created equal? Am J Physiol Heart Circ Physiol. 2014 Aug;307(3):H346-52.

38 Allsopp RC, Chang E, Kashefi-Aazam M, Rogaev EI, Piatyszek MA, Shay JW, et al. Telomere shortening is associated with cell division in vitro and in vivo. Exp Cell Res. 1995 Sep;220(1):194-200.

39 Wang X, Michaelis EK. Selective neuronal vulnerability to oxidative stress in the brain. Front Aging Neurosci. 2010 Mar;2(12):12-3.

40 Prowse KR, Greider CW. Developmental and tissue-specific regulation of mouse telomerase and telomere length. Proc Natl Acad Sci U S A. 1995 May;92(11):4818-22. 